\title{
A NON-HYDROSTATIC TWO-LAYER STAGGERED SCHEME FOR TRANSIENT WAVES DUE TO ANTI-SYMMETRIC SEABED THRUST
}

\author{
S.R. PUDJAPRASETYA \\ Industrial \& Financial Mathematics Research Group, Faculty of Mathematics E Natural \\ Sciences, \\ Institut Teknologi Bandung, \\ Jalan Ganesha 10, Bandung, 40132, Indonesia \\ I. MAGDALENA \\ Industrial \& Financial Mathematics Research Group, Faculty of Mathematics E Natural \\ Sciences, \\ Institut Teknologi Bandung, \\ Jalan Ganesha 10, Bandung, 40132, Indonesia \\ S.S. TJANDRA* \\ Industrial Engineering, Parahyangan Catholic University, \\ Jalan Ciumbeuleuit 94, Bandung 40141
}

Received date

Accepted date

\begin{abstract}
The development of transient waves generated by bottom motion is studied numerically in this work. A non-hydrostatic numerical scheme, based on solving the two dimensional Euler equations using two-layer approximation for the vertical direction, is implemented. The dispersion relation of this scheme is shown to agree with the analytical dispersion relation over a wide range of $k d_{0}$, where $k$ denotes the wave number and $d_{0}$ the characterustic water depth. To ensure that a good balance between non-linearity and dispersion is accommodated by the scheme, the propagation of a solitary wave (undisturbed in shape) was simulated. Our next focus was on the simulation of transient waves generated by bottom motion. After conducting a benchmark test against Hammack's experimental results for downward bottom motion, an anti-symmetric bottom thrust was considered. The resulting transient waves developed different behavior depending on the water depth. Finally, to mimic the December 2004 tsunami, a seabed motion was generated over Aceh bathymetry. This simulation showed that a package of wave trains developed and propagated towards the Aceh coast, and exhibited inter alia the feature of shoreline withdrawal often observed.
\end{abstract}

Keywords: Euler equations; non-hydrostatic pressure; wave generation; wave propagation; tsunami.

* Industrial \& Financial Mathematics Research Group, Faculty of Mathematics \& Natural Sciences, Institut Teknologi Bandung, Jalan Ganesha 10, Bandung, 40132, Indonesia 
2 S.R. Pudjaprasetya et.al.

\section{Introduction}

Seabed disturbances that displace large amounts of water are the primary mechanism for tsunami generation. Thus the sea surface typically deforms in response to a sudden movement of the sea floor caused by a submarine earthquake, and the deformed surface then propagates into the open ocean as a tsunami. Over the ocean depth, the propagation is dispersive (i.e. wave components of different wavelengths travel at different speeds) and the whole process is nonlinear.

The problem of tsunami generation and propagation has deserved careful study. Berry [2005], and Mei et al. [2005] analytically investigated transient waves generated by bottom motion based on linear theory, and numerical investigations have previously been undertaken by many authors.. Dutykh et al. [2007] presented a numerical simulation of waves generated by various types of bottom motion, and Kervella et al. [2007] compared a three-dimensional linear and a nonlinear BEM tsunami generation model. Saito and Furumura [2009] implemented the finite difference method in a three-dimensional simulation of tsunami generation due to sea-bottom deformation. Fuhrman and Madsen [2009] conducted an analytical and numerical study of a high order Boussinesq model to consider the generation and propagation of tsunamis, including the run-up. Experimental studies of tsunami generation were undertaken by Takahashi et al. [1993] and Hatori [1966]. Zhao et al. [2009] used a Boussinesq model to simulate a tsunami wave generated by submarine slumps and slides numerically, and Zhao et al. [2013] studied a potential tsunami generated in the Okinawa Trench and consider the evolution of tsunami waves over continental shelves with gentle slope. Due to different scale of seabed deformation, different waveforms in the near shore regions were found. In this article, we simulate the development of transient waves resulting from an anti-symmetric bottom thrust, using a two-layer non-hydrostatic staggered scheme to solve the Euler equations in two dimensions. After a detailed description of this efficient scheme, we show it leads to typical features associated with a tsunami - viz. its arrival is often preceded by the withdrawal of water from a beach and the first crest arriving may not be the largest.

Our numerical scheme is a variant of the non-hydrostatic staggered approach solving the two-dimensional Euler equations originally proposed by Stelling and Zijlema [2003]. The scheme is based on a conservative staggered scheme for the shallow water equations (SWE) that satisfactorily models long waves in shallow areas - i.e. when $k d_{0} \in[0, \pi / 10]$, where $k$ is the wave number and $d_{0}$ the water depth. Beyond this interval, in the area of dispersive wave, the shallow water model which neglects the dynamic pressure is no longer adequate and the full Euler equations should be incorporated. However, solving the Euler equations requires much heavier computation, particularly if the additional vertical dimension is resolved into small grids. Thus for computational efficiency, numerical algorithm solving the 
Euler equations with few-layer in the vertical direction is of our interest. Here, we focus on a two-layer scheme and conduct several benchmark tests.

In Section 2, the two-layer non-hydrostatic staggered scheme is formulated. The dispersion relation of this scheme is then derived and shown to conform to the analytical dispersion over a wide range of $k d_{0}$, and a solitary wave simulation is performed. In Section 3, we focus on the simulation of transient waves generated by bottom motion. It is notable that our scheme can handle active generation, which mean dynamics of the seafloor displacement can be computed. After simulating the Hammack [1973] benchmark test of free surface wave generated by a downward bottom thrust, an anti-symmetric bottom thrust was considered. In response to the anti-symmetric motion, the free surface developed into a package of wave trains, exhibiting typical tsunami behavior — viz. a tsunami arrival preceded by a withdrawal from the shoreline. For relevance to the 2004 Indian Ocean tsunami, the same anti-symmetric bottom motion was applied to a cross-section of the Aceh bathymetry to simulate an hypothetical tsunami. The simulation generated a wave consisting of a pulse and a trough propagating to the surrounding area. This wave hit the eastern coast starting with a trough, while the waves crashed into the west coast in the form of a pulse. Finally, our conclusions are given in Section 4.

\section{Two-layer non-hydrostatic staggered scheme}

We consider a layer of ideal fluid bounded above by a surface $z=\eta(x, t)$ and below by an impermeable bottom $z=-d(x, t)$. Let $u(x, z, t)$ and $w(x, z, t)$ denote the respective horizontal and vertical fluid velocity components in the two-dimensional Euler equations

$$
\begin{array}{r}
u_{t}+u u_{x}+w u_{z}+g \eta_{x}+q_{x}=0 \\
w_{t}+u w_{x}+w w_{z}+q_{z}=0 \\
u_{x}+w_{z}=0
\end{array}
$$

where the total pressure is decomposed into its hydrostatic and hydrodynamic parts - i.e.

$$
\text { total pressure }=g(\eta-z)+q(x, z, t),
$$

with $q(x, z, t)$ denoting the hydrodynamic pressure.

In our algorithm for integrating the Euler equations $(1,2,3)$, the vertical region is divided into layers with the same thickness. Although better accuracy should be gained if more layers are used, for numerical efficiency a model with only a few layers may be adopted. Indeed, thorough comparison between numerical and analytical results, and also with experimental results obtained by Stelling and Zijlema [2003], suggest that accurate wave characteristics involving dispersion and non-linearity 
4 S.R. Pudjaprasetya et.al.

can be achieved using only two layers. Here we adopt the two-layer scheme, and its formulation is described below.

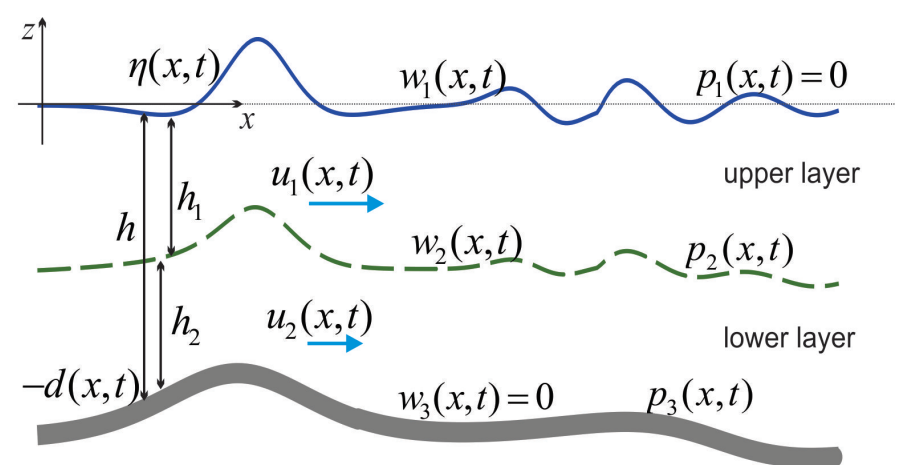

Fig. 1. The staggered grid and positions of the unknowns.

Figure 1 shows a sketch of the fluid domain. The total water depth is $h(x, t)=$ $\eta(x, t)+d(x, t)$; and $z_{k}(x, t)$ for $k=1,2$ and $\left.z_{3}(x, t)=-d(x, t)\right)$ denote the free surface, interface and bottom topography, respectively. The vertical flow region is divided into two layers of the same thickness $h_{1}(x, t)=h_{2}(x, t)=\frac{1}{2} h(x, t)$. Further, we write

$$
w_{k}(x, t) \equiv w\left(x, z=z_{k}, t\right), \quad p_{k}(x, t) \equiv q\left(x, z=z_{k}, t\right), \quad \text { for } \quad k=1,2,3,
$$

and locate the grid points for $w_{k}$ and $p_{k}$, for $k=1,2,3$, along the surface, interface and bottom topography - and systematically set vanishing pressure along the surface $p_{1}=0$ and vanishing vertical velocity along the bottom $w_{3}=0$.

We apply the Keller-box or Preissmann scheme; see e.g. Lam and Simpson [1976], to the linearized equation (2) to yield

$$
\frac{1}{2}\left(\partial_{t} w_{k}+\partial_{t} w_{k+1}\right)=-\frac{p_{k}-p_{k+1}}{h_{k}}, \quad \text { for } k=1,2,
$$

in the upper and lower layer, respectively. Then introducing the depth averaged horizontal velocities for the upper and lower layer as

$$
u_{k}(x, t) \equiv \frac{1}{h_{k}} \int_{\text {layer-k }} u(x, z, t) d z, \quad \text { for } k=1,2,
$$

the respective depth integrated momentum equations are

$$
\partial_{t} u_{k}+u_{k} \partial_{x} u_{k}+g \eta_{x}=-\frac{1}{2}\left(\partial_{x} p_{k}+\partial_{x} p_{k+1}\right), \quad \text { for } k=1,2,
$$


where we approximate the integral using a trapezoidal rule. Applying the Keller-box scheme to approximate the continuity equation (3) for both the upper and lower layers yields

$$
\frac{w_{k}-w_{k+1}}{h_{k}}+\partial_{x} u_{k}=0, \quad \text { for } k=1,2 .
$$

Finally, to determine the water level $\eta(x, t)$ we use the depth integrated continuity equation

$$
h_{t}+\partial_{x}\left(h_{1} u_{1}\right)+\partial_{x}\left(h_{2} u_{2}\right)=0 .
$$

Summarizing, the two-layer non-hydrostatic approximation consists of the seven equations $(6,8,9,10)$.

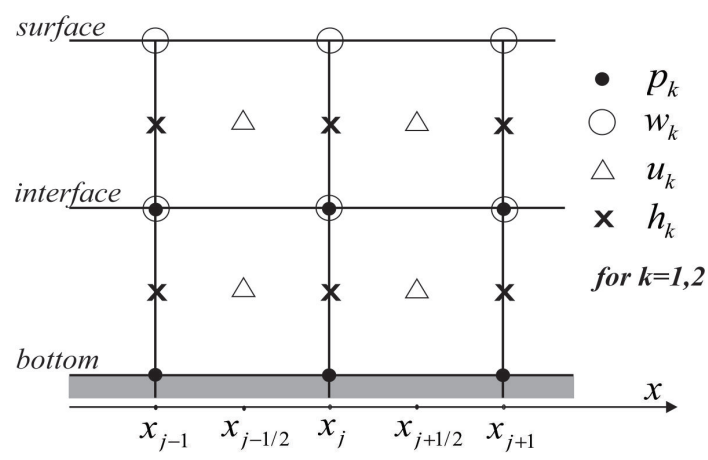

Fig. 2. The staggered grid and positions of the unknowns.

We proceed further with formulating two-layer non-hydrostatic staggered grid scheme as follows. The horizontal $x$-axis is divided in a staggered way, and locations of the unknowns are depicted in Figure 2. Our scheme belongs to the edge-based scheme category, since the dependent variables $p_{2}, p_{3}, w_{1}, w_{2}$ are located at the edge of each cell. Other depth integrated schemes are mostly center-based. In our edgebased scheme the zero pressure condition at the surface and the zero vertical velocity component along the bottom can be obtained accurately. This is actually the key point that makes our two-layer algorithm effective and efficient. The full discrete 
system corresponding to $(6,8,9,10)$ is thus:

$$
\begin{aligned}
& \frac{h_{j}^{n+1}-h_{j}^{n}}{\Delta t}+\frac{{ }^{*} h_{j+\frac{1}{2}}^{n} u_{1, j+\frac{1}{2}}^{n}-{ }^{*} h_{j-\frac{1}{2}}^{n} u_{1, j-\frac{1}{2}}^{n}}{2 \Delta x}+\frac{{ }^{*} h_{j+\frac{1}{2}}^{n} u_{2, j+\frac{1}{2}}^{n}-{ }^{*} h_{j-\frac{1}{2}}^{n} u_{2, j-\frac{1}{2}}^{n}}{2 \Delta x}=(11) \\
& \frac{u_{1, j+\frac{1}{2}}^{n+1}-u_{1, j+\frac{1}{2}}^{n}}{\Delta t}+\left.\left(u_{1} \partial_{x} u_{1}\right)\right|_{j+\frac{1}{2}} ^{n}+g \frac{\eta_{j+1}^{n+1}-\eta_{j}^{n}}{\Delta x}=-\frac{p_{2, j+1}^{n+1}-p_{2, j}^{n+1}}{2 \Delta x} \\
& \frac{u_{2, j+\frac{1}{2}}^{n+1}-u_{2, j+\frac{1}{2}}^{n}}{\Delta t}+\left.\left(u_{2} \partial_{x} u_{2}\right)\right|_{j+\frac{1}{2}} ^{n}+g \frac{\eta_{j+1}^{n+1}-\eta_{j}^{n}}{\Delta x}=-\frac{p_{2, j+1}^{n+1}-p_{2, j}^{n+1}}{2 \Delta x}-\frac{\left.p_{3, j+1}^{n+1}-p_{3, j}^{n+1}\right)}{2 \Delta x} \\
& \frac{1}{2} \frac{w_{1, j}^{n+1}-w_{1, j}^{n}}{\Delta t}+\frac{1}{2} \frac{w_{2, j}^{n+1}-w_{2, j}^{n}}{\Delta t}=-2 \frac{0-p_{2, j}^{n+1}}{h_{j}^{n+1}} \\
& \frac{1}{2} \frac{w_{2, j}^{n+1}-w_{2, j}^{n}}{\Delta t}=-2 \frac{p_{2, j}^{n+1}-p_{3, j}^{n+1}}{h_{j}^{n+1}} \\
& w_{1, j}^{n+1}-w_{2, j}^{n+1}+h_{j}^{n+1} \frac{u_{1, j+\frac{1}{2}}^{n+1}-u_{1, j-\frac{1}{2}}^{n+1}}{2 \Delta x}=0 \\
& w_{2, j}^{n+1}-0+h_{j}^{n+1} \frac{u_{2, j+\frac{1}{2}}^{n+1}-u_{2, j-\frac{1}{2}}^{n+1}}{2 \Delta x}=0
\end{aligned}
$$

Here we have taken $h_{1}=h_{2}=h / 2$, zero pressure along the surface $\left(p_{1}=0\right)$, and zero vertical velocity along the bottom $\left(w_{3}=0\right)$. In $(11)$ the notation ${ }^{*}$ means the upwind approximation

$$
{ }^{*} h_{j+\frac{1}{2}}^{n}= \begin{cases}h_{j}^{n}, & \text { if }\left(u_{1, j+\frac{1}{2}}^{n}+u_{2, j+\frac{1}{2}}^{n}\right)>0 \\ h_{j+1}^{n}, & \text { if }\left(u_{1, j+\frac{1}{2}}^{n}+u_{2, j+\frac{1}{2}}^{n}\right)<0 \\ \max \left(\eta_{j}, \eta_{j+1}\right)+\min \left(d_{j}, d_{j+1}\right), & \text { if }\left(u_{1, j+\frac{1}{2}}^{n}+u_{2, j+\frac{1}{2}}^{n}\right)=0\end{cases}
$$

Moreover, the advection terms $u_{k} \partial_{x} u_{k}$, for $k=1,2$ are calculated using the momentum conservative approximation described in Stelling and Duinmeijer [2003].

The hydrodynamic terms $p_{2}, p_{3}$ in (12-15) are calculated using a predictorcorrector method as follows. For each time step, the $h^{n+1}$ are computed from (11). The predicted values of $u_{1}, u_{2}$ are then computed from $(12,13)$ on setting the righthand side to zero (omitting hydrodynamic terms), and these predicted values (indicated with ^) are corrected using

$$
\begin{aligned}
& u_{1, j+\frac{1}{2}}^{n+1}=\hat{u}_{1, j+\frac{1}{2}}-\frac{\Delta t}{2 \Delta x}\left(p_{2, j+1}^{n+1}-p_{2, j}^{n+1}\right), \\
& u_{2, j+\frac{1}{2}}^{n+1}=\hat{u}_{2, j+\frac{1}{2}}-\frac{\Delta t}{2 \Delta x}\left(p_{2, j+1}^{n+1}-p_{2, j}^{n+1}+p_{3, j+1}^{n+1}-p_{3, j}^{n+1}\right) .
\end{aligned}
$$

In this correction step, the dynamic pressure values $p_{2}^{n+1}, p_{3}^{n+1}$, are needed, and they are calculated first. Now adding two times (16) to (17) provides the equation

$$
w_{1, j}^{n+1}+w_{2, j}^{n+1}+h_{j}^{n+1} \frac{u_{1, j+\frac{1}{2}}^{n+1}-u_{1, j-\frac{1}{2}}^{n+1}}{2 \Delta x}+h_{j}^{n+1} \frac{u_{2, j+\frac{1}{2}}^{n+1}-u_{2, j-\frac{1}{2}}^{n+1}}{\Delta x}=0,
$$


and substituting $(14,15,19,20)$ into $(21)$ results in a linear system of equations for $p_{2, j}^{n+1}, p_{3, j}^{n+1}$ that can be solved using an iterative method. In summary, our computational procedure is as follows.

1. Compute $h^{n+1}$ from (11).

2. Compute the predicted values of $\hat{u}_{1}, \hat{u}_{2}$ using $(12,13)$ with zero right hand sides.

3 . Solve the linear system for $p_{1}^{n+1}, p_{2}^{n+1}$ by successive over-relaxation method.

4. Compute the corrected values of $u_{1}^{n+1}, u_{2}^{n+1}$ using $(19,20)$, respectively.

5. Compute $w_{2}^{n+1}$ using (15), and $w_{1}^{n+1}$ using (14).

It is notable that the staggered scheme of the SWE hydrostatic numerical model is just the predictor step; for every time step we compute $h^{n+1}$ and $u_{1}^{n+1}, u_{2}^{n+1}$ from step 1 and step 2, respectively. For simulation that contains dry areas, the following wet-dry procedure should be implemented; if $h^{n+1}$ is less than a small positive number (which means the corresponding cell is dry enough), step 2 is replaced by simply set $u_{1}^{n+1}$ and $u_{2}^{n+1}$ to zero. Further details on this staggered scheme can be found in Pudjaprasetya and Magdalena [2014]. This scheme will be used later in the simulation of waves propagate towards Aceh coast that involves moving shoreline.

\subsection{Dispersion relation}

We now discuss the dispersion relation of the discrete equations. Substituting

$$
\left(\begin{array}{l}
h_{j}^{n} \\
u_{k, j+1 / 2}^{n} \\
w_{k, j}^{n} \\
p_{k, j}^{n}
\end{array}\right)=\left(\begin{array}{l}
H \\
U_{k} e^{i \Delta x / 2} \\
W_{k} \\
P_{k}
\end{array}\right) e^{i(k j \Delta x-\omega n \Delta t)}
$$

into (11-17) yields a system of linear equations with seven unknowns $H, U_{k}, W_{k}, P_{k}$, for $k=1,2$. After some algebra, we can simplify the coefficient matrix as follows:

$$
\left(\begin{array}{ccccccc}
-i W & \frac{i d_{0} K}{2 E} & \frac{i d_{0} K}{2 E} & 0 & 0 & 0 & 0 \\
i E g K & -i W & 0 & 0 & 0 & \frac{i E K}{2} & 0 \\
i E g K & 0 & -i W & 0 & 0 & \frac{i E K}{2} & \frac{i E K}{2} \\
0 & \frac{i d_{0} k}{2} & 0 & 1 & -1 & 0 & 0 \\
0 & 0 & \frac{i d_{0} K}{2} & 0 & 1 & 0 & 0 \\
0 & 0 & 0 & -i W & 0 & -\frac{8 E}{d_{0}} & \frac{4 E}{d_{0}} \\
0 & 0 & 0 & 0 & -i W & \frac{4 E}{d_{0}} & -\frac{4 E}{d_{0}},
\end{array}\right)
$$

with $E=e^{i \omega \Delta t / 2}, W=\frac{2 \sin (\omega \Delta t / 2)}{\Delta t}, K=\frac{2 \sin (k \Delta x / 2)}{\Delta x}$. The determinant of this coefficient matrix is

$$
\frac{1}{16} \frac{i}{d_{0}^{2}} E^{2} W\left(d_{0}^{4} K^{4} W^{2}-16 d_{0}^{3} g K^{4}+96 d_{0}^{2} K^{2} W^{2}-256 d_{0} g K^{2}+256 W^{2}\right),
$$


and we have a non-trivial solution to the system of linear equations when the determinant is zero - producing the dispersion relation of the discrete scheme

$$
\left(\frac{\omega}{\omega_{0}}\right)^{2}=\frac{16\left(d_{0}^{2} k^{2}+16\right)}{d_{0}^{4} k^{4}+96 d_{0}^{2} k^{2}+256}, \quad \text { with } \quad \omega_{0}^{2} \equiv g k^{2} d_{0},
$$

on invoking $\lim _{\Delta t \rightarrow 0} W=\omega$ and $\lim _{\Delta x \rightarrow 0} K=k$. The ratio $\left(\omega / \omega_{0}\right)^{2}$ is plotted together with the exact dispersion

$$
\left(\frac{\omega_{\text {exact }}}{\omega_{0}}\right)^{2}=\frac{\tanh \left(k d_{0}\right)}{k d_{0}}
$$

in Figure (3), showing that the dispersion relation of our discrete model closely approximates the exact analytical dispersion relation (26) for a wide range of $k d_{0}$. Indeed, for $0<k d_{0}<7$ the numerical dispersion relation (25) approximates the analytical dispersion (26) with an error less than $1.1 \%$.

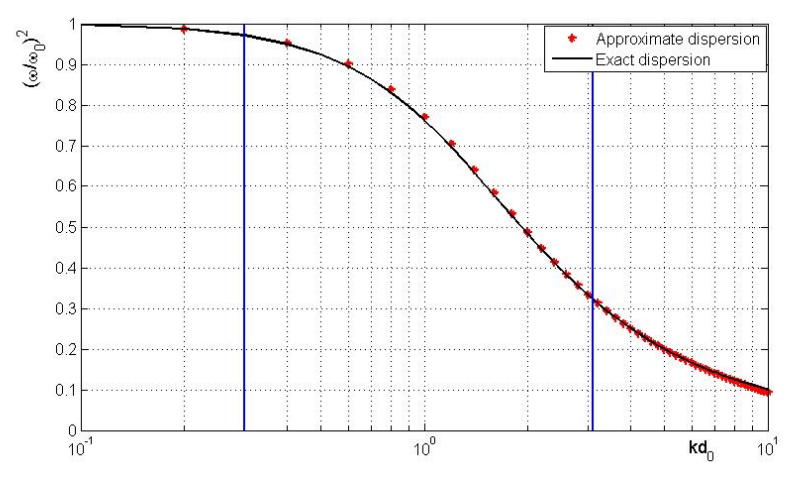

Fig. 3. Dispersion relation (25) in comparison with exact dispersion relation (26).

We next present a test case of standing wave simulation in a closed basin. In a computational domain $[0, L=10 \mathrm{~m}]$, an initial monochromatic wave $\eta(x, 0)=$ $0.1 \cos k x$ with $k=\frac{\pi}{10}$, over a water depth of $d_{0}=10 \mathrm{~m}$ is considered. This is a dispersive wave since $k d_{0}=\pi$, but it is almost linear since the amplitude to depth ratio for this wave is small. The exact solution from linear wave theory is

$$
\eta(x, t)=0.05\left(\cos \left(\frac{\pi}{10}(x-c t)+\cos \left(\frac{\pi}{10}(x+c t)\right)\right),\right.
$$

with the wave speed $c=\omega / k$, and frequency $\omega$ given in (26). For the computation, we took $\Delta x=0.05, \Delta t=0.01$. The numerical wave signal at $x=17.5 \mathrm{~m}$ and the exact solution are plotted in Figure 4. Wave signals calculated using the hydrostatic SWE model and the two-layer non-hydrostatic model are plotted in Figure 4 (top) and (bottom), respectively. The hydrostatic model produces an incorrect wave frequency $\omega=3.14 \mathrm{sec}^{-1}$, whereas the two-layer non-hydrostatic model predicts a 
wave frequency $\omega=1.795 \mathrm{sec}^{-1}$ near the exact analytical frequency $\omega_{\text {exact }}=1.77$ $\sec ^{-1}$.
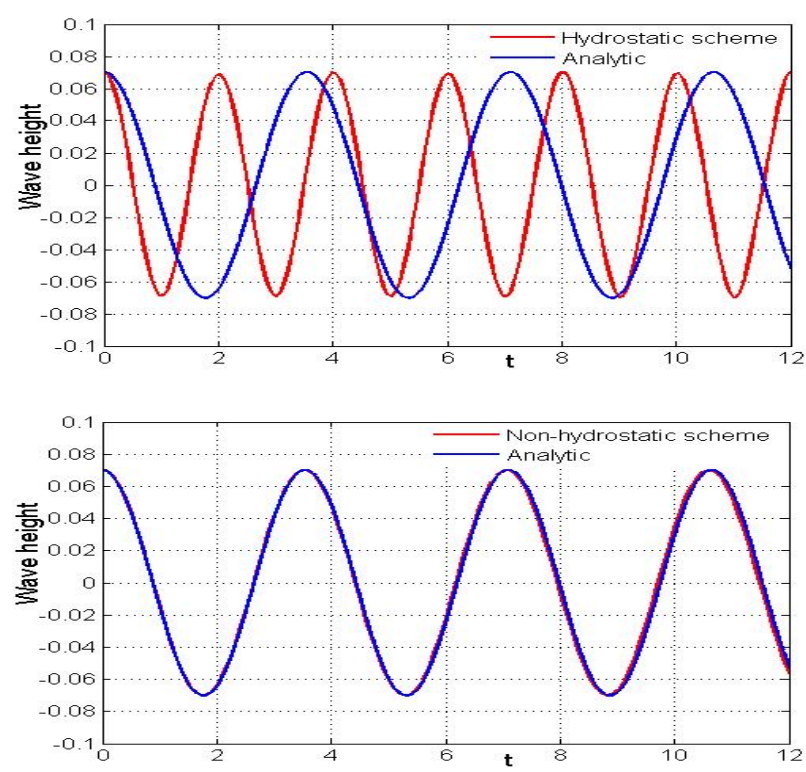

Fig. 4. Wave signal at $x=17.5 \mathrm{~m}$ (top) calculated using SWE hydrostatic model, (bottom) calculated using non-hydrostatic model.

\subsection{Solitary wave simulation}

Our second test of the non-hydrostatic scheme was to simulate a solitary wave, propagating undisturbed in shape with constant velocity. Let $A$ be the wave amplitude, $d_{0}$ the water depth, and $\lambda$ the wave speed. The exact solitary wave is given by

$$
\eta(x, t)=A \operatorname{sech}^{2} \xi(x-\lambda t), \quad \lambda=\sqrt{g\left(d_{0}+A\right)}, \quad \xi=\sqrt{\frac{3 A}{4 d^{2}(d+A)}} .
$$

In a computational domain $[0,1000]$ with grid partition $\Delta x=1, \Delta t=0.1$, the initial solitary wave with parameters $A=1$ and $d_{0}=10$ was adopted. The calculated solitary waves at subsequent times are plotted together with the exact solitary waves in Figure 5, showing that our non-hydrostatic numerical scheme can calculate a solitary wave that closely conforms to the analytical solitary wave over a long period of time. The good agreement with the analytical solitary wave indicates that our scheme can balance the non-linearity and dispersion correctly. 


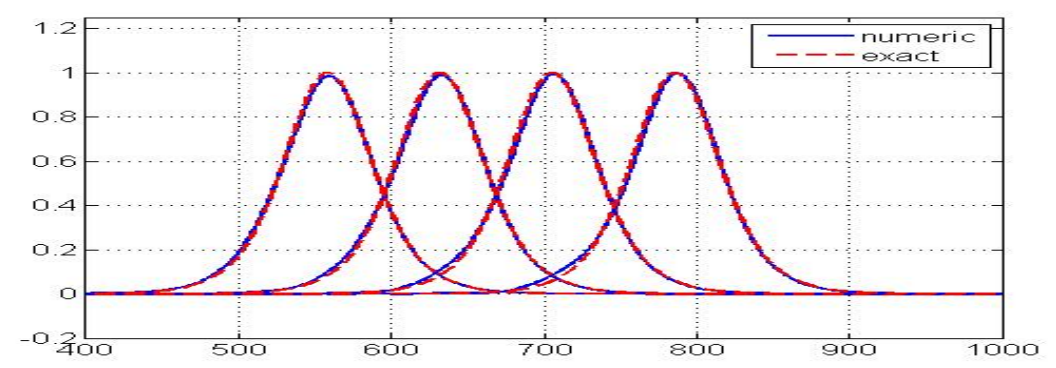

Fig. 5. Numerical solitary wave together with the exact solitary wave at subsequent times $t=$ $0,7,14,21 \mathrm{sec}$

\section{Transient waves generated by seabed thrusts}

A two-layer non-hydrostatic scheme was used to simulate the development of transient waves generated by various seabed thrusts. It is notable that our scheme can handle active generation, in which a time-dependent bottom motion $-d(x, t)$ can be incorporated. To be precise, the term $h_{j}^{n}$ in (11) is in fact $h\left(x_{j}, t^{n}\right) \equiv$ $\eta\left(x_{j}, t^{n}\right)+d\left(x_{j}, t^{n}\right)$.

\subsection{Downwards bottom thrust}

The main experimental benchmark test for tsunami generation scheme is the work by Hammack [1973]. Hammack's conducted experiments in a very long wave tank of length $31.6 \mathrm{~m}$ and width $39.4 \mathrm{~cm}$. In the downward bottom experiment, a section of the bed fell to a maximum displacement $\zeta_{0}$ in an asymptotic manner, governed by

$$
d(x, t)=d_{0}-\zeta_{0}\left(1-e^{-\alpha t}\right) H\left(b^{2}-x^{2}\right),
$$

where $H$ denotes the Heaviside step function. A characteristic time $t_{c}$ may be defined as the time for the bottom thrust to reach $2 / 3$ of the maximum displacement $\zeta_{0}$ - cf. Figure 6 (right). The parameter $t_{c}$ can be adjusted to simulate various types of motion (impulsive, intermediate or slow), but here we focus on impulsive motion.

We mimicked Hammack's downward bottom thrust experiment, where the undisturbed water depth was $d_{0}=5 \mathrm{~cm}$ and the downward section had dimensions $b=61$ $\mathrm{cm}$ and $\zeta_{0}=-0.5 \mathrm{~cm}$. Our computation was conducted in a computational domain $[0, L]$ using the spatial and time steps $\Delta x=b / 12 \mathrm{~cm}$ and $\Delta t=0.004 \mathrm{sec}$. A fully reflecting wall was prescribed at the right and left computational boundaries by taking $u(0, t)=0, u(L, t)=0$, and the simulations were stopped before any reflections occurred. As a result of the downward bottom motion, the water surface drops to a maximum displacement of $-\zeta_{0}$ - and after reaching this maximum the surface 

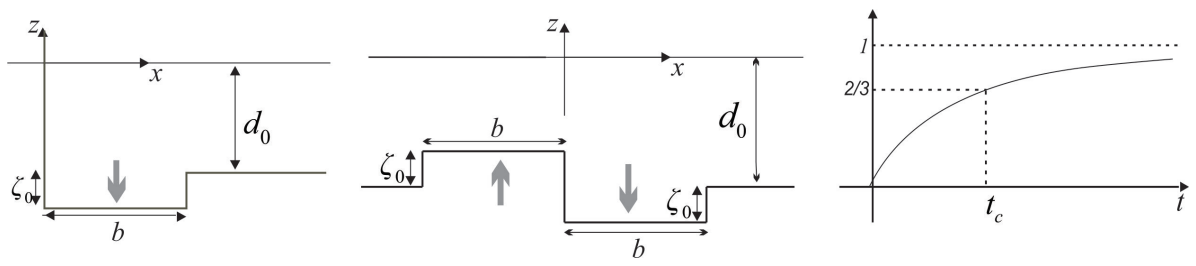

Fig. 6. Bed deformation model: (left) a downwards bottom thrust, (middle) an anti-symmetric bottom thrust, (right) the exponential time-dependent function $\left(1-e^{-\alpha t}\right)$ and the characteristic time $t_{c}$.

returns to the still water level, except for an oscillatory tail.
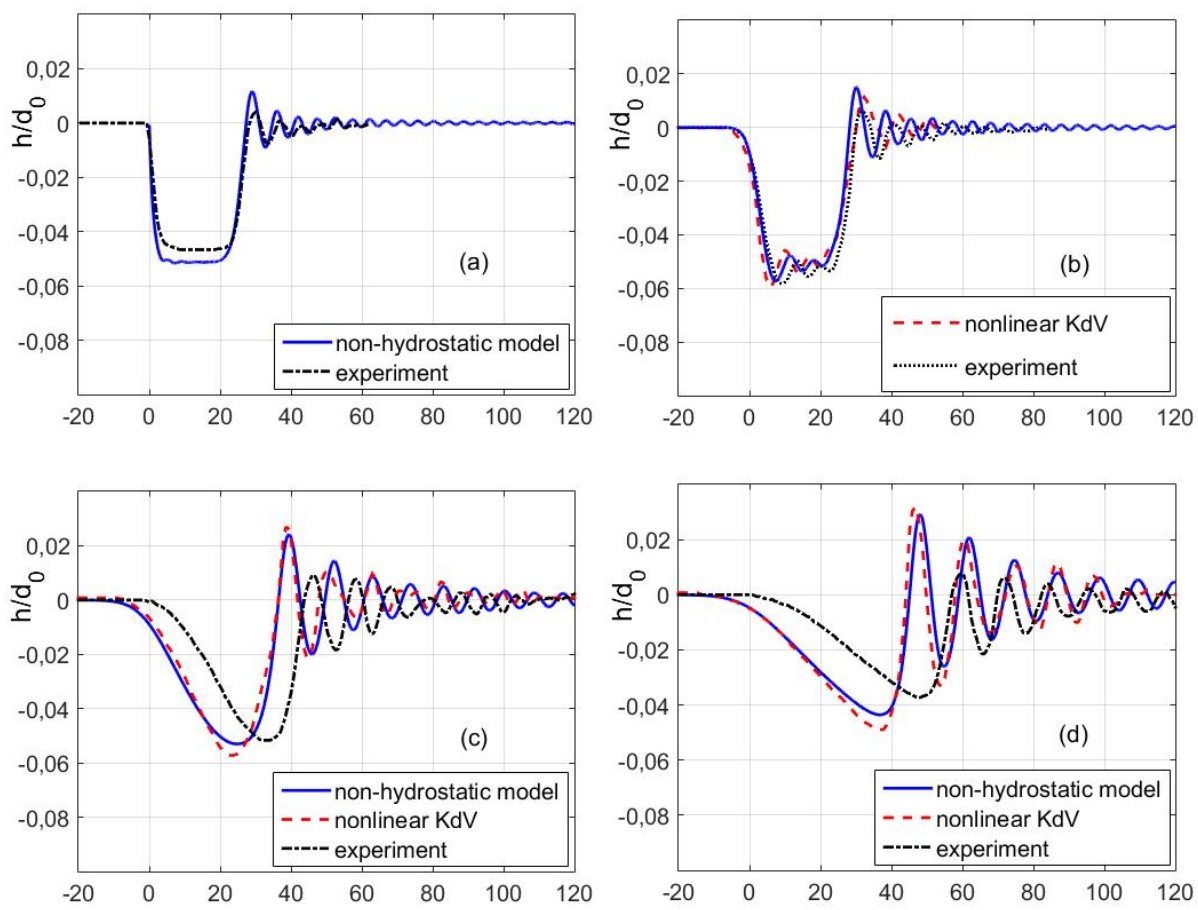

Fig. 7. Time series of surface elevation $\eta / d_{0}$ w.r.t $t \sqrt{g / d_{0}}-(x-b) / d_{0}$ resulting from a downward bottom motion. The waves were recorded at locations (a) $(x-b) / d_{0}=0$, (b) 20, (c) 180, (d) 400 .

Figure 7 presents a time series of the surface elevation computed using our non-hydrostatic scheme, in comparison with Hammack's results. The generated free surface flow was recorded at the four locations $(x-b) / d_{0}=0,20,180,400$, and plotted against the shifted time variable $t \sqrt{g / d_{0}}-(x-b) / d_{0}$. The results show that our non-hydrostatic numerical scheme can accommodate bottom motion. The 
generated wave in Figure 7 (a) is in agreement with Hammack's experimental wave. Simulation at later times, when the main wave has propagated into the domain, our numerical wave signal nicely conform the experimental and numerical results of KdV model, both are taken from Hammack [1973]. Further comparisons with various experiments of Hammack [1973], and also with other numerical models in Lynett and Liu [2002] and Fuhrman and Madsen [2009], can be found in Pudjaprasetya and Tjandra [2014].

\subsection{Anti-symmetric bottom thrust on a constant topography}

Next, we relaxed the hard wall left boundary and observed the development of transient waves resulting from an anti-symmetric bottom motion. In the d'Alembert solution, the initial one-dimensional wave splits into two waves of the same form, one propagating to the left and the other to the right. The linear non-dispersive model predicts the wave shape to be the same as the initial displacement, which may evolve to different waveforms depending on water depth. For tsunami propagation, it is important to know how the waves deform under a fully nonlinear dispersive model, since it determines tsunami feature that approaches a coastline.

Our first investigation used the analytical formula of transient waves developed by a sudden tilting of the bottom reported in Mei et.al. [2005]. We considered an anti-symmetric bottom motion

$$
d(x, t)=d_{0}-\zeta_{0} H(t)(H(x+b)-2 H(x)+H(x+b)),
$$

where $H$ denotes the Heaviside function and $d_{0}$ the initial constant depth. For this anti-symmetric bottom thrust (30), the resulting leftward and rightward propagating waves are symmetric with respect to the origin. The explicit formula for the rightward propagating wave derived from the linearized model is

$$
\zeta(x, t)=\tilde{D}(0)\left(\frac{2}{\sqrt{g h} h^{2} t}\right)^{\frac{2}{3}} A i^{\prime}\left(\left(\frac{2}{\sqrt{g h} h^{2} t}\right)^{\frac{1}{3}}(x-\sqrt{g h} t),\right.
$$

with

$$
\tilde{D}(0)=-\int_{-\infty}^{\infty} x H_{0}^{\text {odd }}(x) d x, \quad H_{0}^{\text {odd }}(x)=\frac{d d}{d x} .
$$

The above formulation corresponds to $\omega^{2} \approx g k\left(k d_{0}-\frac{1}{3} d_{0}^{3} k^{3}\right)$, a two-term approximation of the well known water wave dispersion relation $\omega^{2}=g k \tanh k d_{0}$, and we expect (31) to hold for intermediate or deep-water areas. Moreover, the main feature of the resulting surface wave is given by $A i^{\prime}(z)$ as depicted in Figure 8. This encoutaged us to study transient wave development resulting from an antisymmetric bottom motion.

We conducted further simulations using the bottom motion (30) over a flat depth $d_{0}$, where the computation domain was $[0,120 \mathrm{~km}]$ and the bottom thrust 


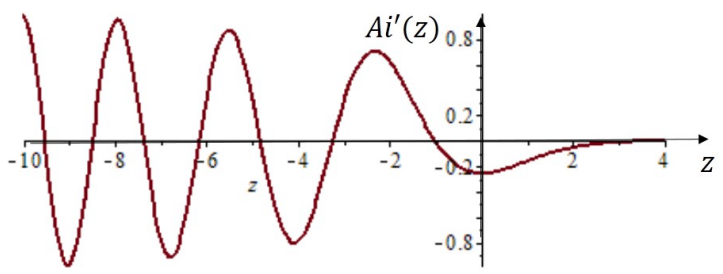

Fig. 8. The curve of $A i^{\prime}(z)$ as a function of $z$, which shows a train of waves with increasing amplitude led by a negative wave.

was located at $40 \mathrm{~km}$. We took the parameters $\zeta_{0}=10 \mathrm{~m}, b=1919.4 \mathrm{~m}$ for the anti-symmetric bottom thrust, $t_{c}=2 \mathrm{sec}$, and the gravity $g=9.8 \mathrm{~m} / \mathrm{sec}^{2}$; and $\Delta x=383.9 \mathrm{~m}$ and $\Delta t=0.2 \mathrm{sec}$ for the spatial and time steps. These parameters were also used in the simulation over Aceh bathymetry, given in the next subsection. Thus the anti-symmetric bottom (30) was set in motion, generating a propagating surface wave. In the first two simulations, the bottom was flat: $d_{0}=600 \mathrm{~m}$ (shallow water depth) and $d_{0}=1100 \mathrm{~m}$ (intermediate depth).
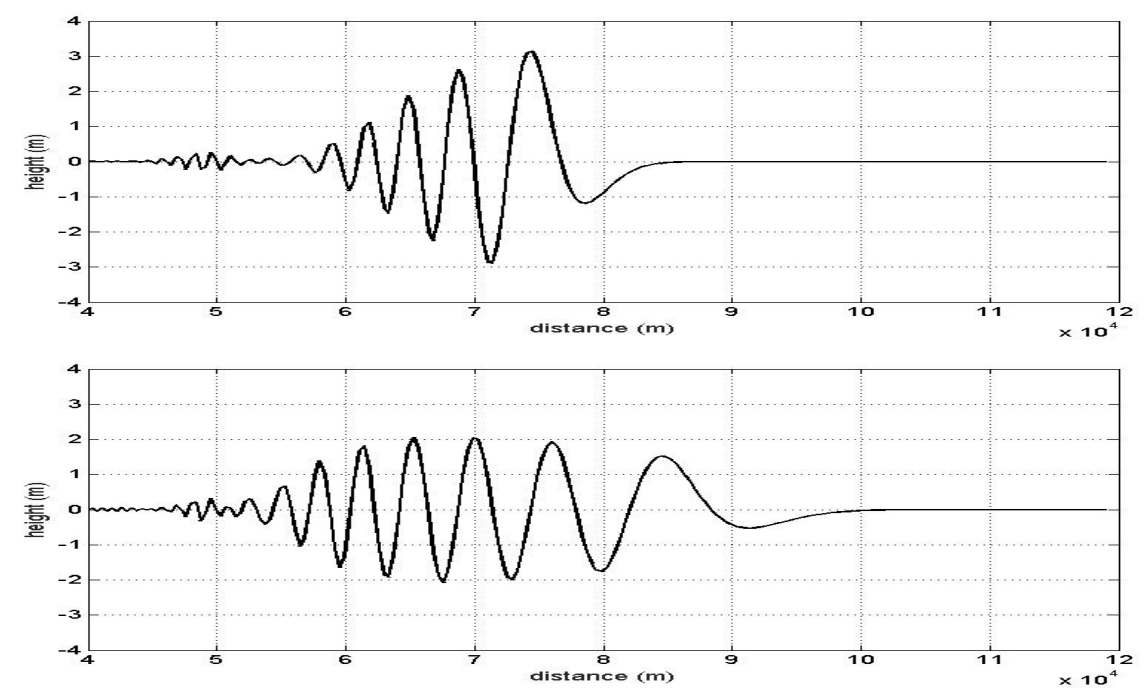

Fig. 9. The right running part of the transient wave after 8 minutes of propagation over (top) a shallow depth $d_{0}=600 \mathrm{~m}$, (bottom) an intermediate depth $d_{0}=1100 \mathrm{~m}$.

In Figure 9 the development of transient waves over a flat depth is presented. In response to the anti-symmetric bottom thrust, in both Figures 9 (top) and (bottom) a package of wave trains developed preceded by a negative wave front, but quite different features were observed. In the case of the intermediate water depth, due 
to a stronger dispersive effect the developed wave train package has features like the $A i^{\prime}$ function, where the first pulse is followed by the second and third pulses, etc.. In this simulation, the amplitude of the third pulse is larger that the first two. However, the same bottom thrust at a shallower depth develops different tsunami wave trains. In this case, the amplitude of the first wave is the largest, and its maximum amplitude exceeds the maximum amplitude in the intermediate depth case — cf. Figure 9 (top).

On their way to the beach, the wave packages passing over a continental shelf undergo shoaling effects where the velocity and wavelength decreases and their amplitude increases, but the main features stay the same, with the rightward propagating wave packages is preceded by a leftward propagation. This may explain a phenomenon often reported - viz. that the arrival of a tsunami arrival is preceded by the ocean receding from the shoreline. Furthermore, depending on the depth, different types of wave packages may develop. Thus over a relatively shallow region the leading wave has the largest amplitude, whereas over a relatively deep region the the second or the third wave has the largest amplitude, as in the 1868 tsunami in Africa and the 1946 tsunami in Hilo, Hawaii — cf. Bryant [2008]. It was reported that many people were killed by the third wave, which was higher that the preceding two.

\subsection{Anti-symmetric bottom thrust over Aceh bathymetry}

The anti-symmetric bottom thrust was also applied to a cross-sectional bathymetry representative of Aceh, Sumatra, Indonesia. Our computation was directed to a simulation of a typical tsunami surface wave propagating over the Indian Ocean towards the coastline.

The Indian Ocean bathymetry near Aceh obtained from NOAA, with one minute resolution, is displayed in Figure 10 (left). The hypocentre of the 2004 Indian Ocean earthquake is indicated as a black circle, and the cross-section $\left(95.0278^{\circ} \mathrm{E}\right.$, $\left.3.2335^{\circ} \mathrm{N}\right)-\left(96.6583^{\circ} \mathrm{E}, 3.6959^{\circ} \mathrm{N}\right)$ connects this hypocentre to the nearest Aceh coast. The bathymetry along this cross-section is depicted in Figure 10 (right), and using this bathymetry we simulated a transient wave developed by the antisymmetric bottom thrust.

Parameters for the bottom motion used here were the same as in the previous runs, and the resulting transient wave is shown in Figure 11. If we follow the rightward propagating wave train: the wave approaching the eastern coast is preceded by a wave trough. This means that the tsunami arrival along Aceh beaches was preceded by a drawback from the shoreline, exposing a normally submerged seabed, which Bryant [2008] reported was not observed along the African or any other eastfacing coast. Following the right running waves, they will arrive on a relatively 

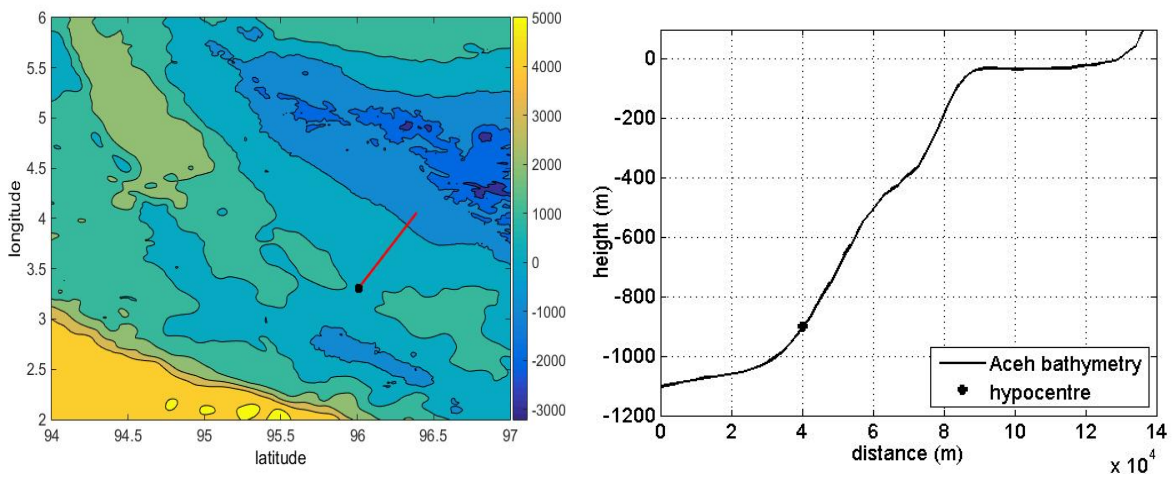

Fig. 10. Indian Ocean bathymetry near Aceh with the color bar representing water depth $(\mathrm{m})$ (left) and cross-section (right) at $\left(95.0278^{\circ} \mathrm{E}, 3.2335^{\circ} \mathrm{N}\right)-\left(96.6583^{\circ} \mathrm{E}, 3.6959^{\circ} \mathrm{N}\right)$.

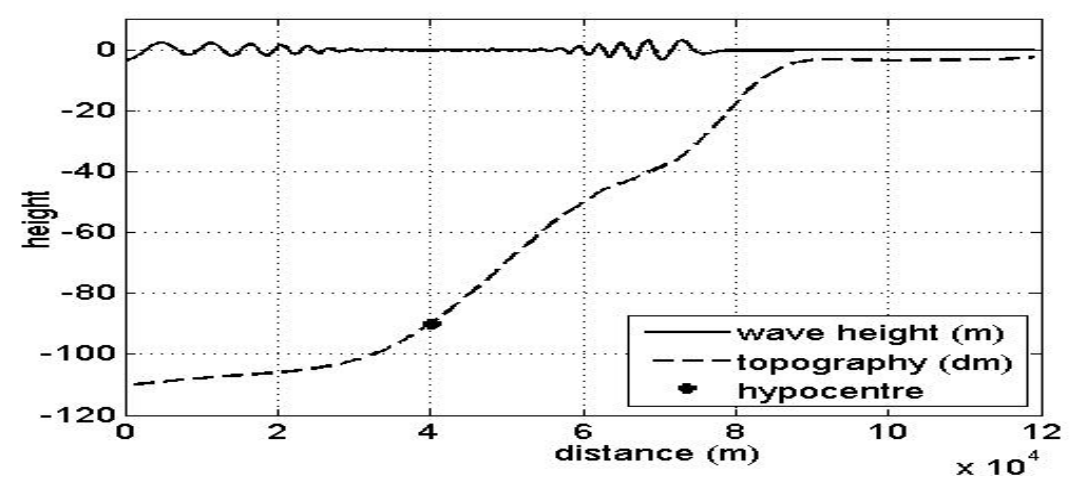

Fig. 11. Transient wave after 8 minutes of propagation over cross-sectional Aceh bathymetry.

shallow water area. Computation is conducted using the SWE hydrostatic model including wet-dry procedure, and the wave profiles at subsequent times are plotted in Figure 12, where three waves are clearly visible. Of these waves, the second that appears to have the largest amplitude must be expected to hit the coast with the largest energy. In passing, we note this hypothetical tsunami simulation may not be realistic, for which detailed data related to the earthquake and an accurate model for subduction slab are needed.

\section{Conclusions}

A non-hydrostatic numerical scheme to solve the two-dimensional Euler equations using a two-layer approach was considered. For $k d_{0} \in[0,7]$, the dispersion relation of the scheme agrees with the exact dispersion relation with an error less than $1.1 \%$. 

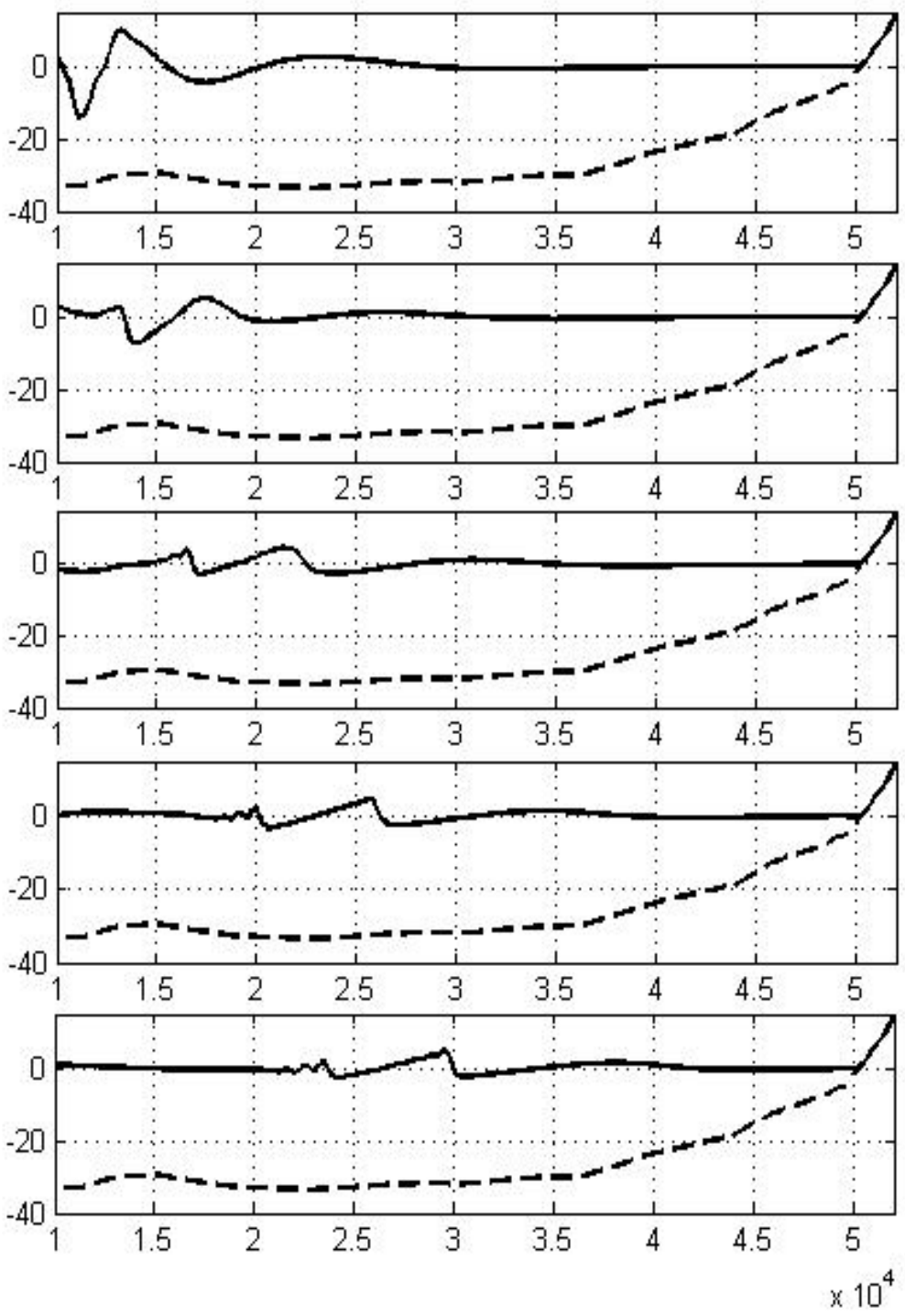

Fig. 12. Wave profiles over a shallow water area of Aceh coast at subsequent times.

Moreover, the scheme produced a negative wave that conforms with Hammack's experimental test, in response to a downward bottom thrust. For an anti-symmetric seabed thrust, the simulated wave developed a package of free surface wave trains preceded by a negative wave front, which may explain the observed phenomenon 
that an incoming tsunami is preceded by an apparent ocean withdrawal from the shoreline due to a wave trough, which is then followed by a pulse that bring about a strong impulsive flooding. In some cases the second or the third wave pulse may have larger amplitude than the first. Finally, we emphasise that the precise form of the tsunami wave train approaching a shore strongly depends on the precise bottom motion and the inshore topography.

Acknowledgement: The authors would like to acknowledge the support of the Ensemble Estimation of Flood Risk in a Changing Climate project funded by The British Council through their Global Innovation Initiative. The support from Riset Desentralisasi 2016 No. 0056/E3.2/LT/2016 is acknowledged.

\section{Journal reference:}

Berry, M.V. [2005] "Tsunami Asymptotics," in New Journal of Physics 7, 129.

Bryant, E. [2008], Tsunami the underrated hazard $2^{\text {nd }}$ ed., (Springer, Praxis Publishing Chichester, UK).

Dutykh, D. and Dias, F. [2007] "Water waves generated by a moving bottom," in Tsunami and Nonlinear Waves, 65-96.

Fuhrman, D.R. and Madsen, P.A. [2009], "Tsunami generation, propagation, and run up with high order Boussinesq model," in Coastal Engineering 56, 747-758.

Hammack, J. L. [1973], "A note on tsunamis: their generation and propagation in an ocean of uniform depth," in J. Fluid Mech. 4, 769-799.

Hatori, T. [1966], "Vertical displacement in a tsunami source area and the topography of the sea bottom," in Bull. Earthquake Res. Inst. Tokyo Univ., 44, 14491464.

Kajiura, K. [1970], "Tsunami source, energy and the directivity of wave radiation", Bull. Earthq. Res. Ins., 48, 835869.

Keller, J. B. [1961], "Tsunamis-Water waves produced by earthquakes, Proceedings of Tsunami Hydrodynamics Conference, Inst. Geophs., University of Hawaii 24, pg 154-166.

Kervella, Y., Dutykh, D., Dias, F. [2007], "Comparison between three-dimensional linear and nonlinear tsunami generation models," in Theor. Comput. Fluid Dyn. 21 245-269.

Lam DCL, Simpson RB. [1976], "Centered differencing and the box scheme for diffusion convection problems," in Journal of Computational Physics 22486500.

Lynett, P., Liu and P.L.F. [2002], "A numerical study of submarine-landslidegenerated waves and codes", in ITS Proceedings Session 7, No. 7-13.

Maeda, T. and Furumura, T. [2011], "FDM simulation of seismic waves, ocean acoustic waves, and tsunamis based on tsunami-coupled equations of motion," 
in Pure and App. Geo..

Magdalena, I. [2015], "An Efficient Two-Layer Non-hydrostatic Numerical Model for Waves Propagation", Ph.D. thesis, Mathematics Dept., Institut Teknologi Bandung, Bandung.

Mei, C. C., Stiassnie, M., Yue, D. P. [2005] Theory and Applications of Ocean Surface Waves, (Advanced series on Ocean Engineering).

Nalbant, S., Steacy, S., Sieh, K., Natawidjaja, D., and McCloskey, J. [2009], "Seismology: Earthquake risk on the Sunda trench," Nature Vol. 435 (7043), 756757.

Pudjaprasetya, S.R. and Magdalena, I. [2014], "Momentum Conservative Scheme for Shallow Water Flows," East Asian Jour. on App. Math. (EAJAM), 4 (2), 152-165.

Pudjaprasetya, S.R. and Tjandra, S.S. [2014] "A Hydrodynamic Model for dispersive waves generated by bottom motion," Springer Proc. in Math. ES Statistic, FVCA'

Saito, T. and Furumura, T. [2009], "Three-dimensional tsunami generation simulation due to sea-bottom deformation and its interpretation based on the linear theory," Geophys. J. Int. 178, 877-888.

Stelling, G.S. and Duinmeijer, S.P.A., [2003] "A staggered conservative scheme for every Froude number in rapidly varied shallow water flows," International Journal For Numerical Methods in Fluids A 43, 1329-1354.

Stelling, G.S. and Zijlema, M. [2003] "An accurate and efficient finite difference algorithm for non hydrostatic free surface flow with application to wave propagation," International Journal For Numerical Methods in Fluids 43, 1-23.

Takahashi, T., F. Imamura, and N. Shuto [1993], "Numerical simulation of topography change due to tsunamis", paper presented at IUGG/IOC International Tsunami Symposium, Int. Union of Geod. and Geophys., Wakayama, Japan.

Todorovska M.I. and Trifunac M.D. [2001], "Generation of tsunamis by a slowly spreading uplift of the sea-floor. Soil Dynamics and Earthquake Engineering 21:151167 un-up," Proc. R. Soc. Lond. A 458, 2885-2910.

Tjandra, S.S. and Pudjaprasetya, S.R. [2015], "A non-hydrostatic numerical scheme for dispersive waves generated by bottom motion," Wave Motion 57, 245-256.

Ursell, F. [1953], "The long-wave paradox in the theory of gravity waves," Proc. Camb. Phil. Soc. 49, 685-694.

Watts, P., Grilli, S.T., Imamura, F. [2001], "Coupling of tsunami generation and propagation," ITS 2001 Proceedings, Session 7, No 7-13, 811-824.

Zhao, X., Wang, BL., Liu H. [2009], "Modelling the submarine mass failure induced tsunamis by Boussinesq equations," Journal of Asian Earth Sciences, No. 36, $47-55$.

Zhao X., Liu H., Wang BL. [2013], "Evolvement of tsunami waves on the continental shelves with gentle slope in the China Seas," Theor. Appl. Mech. Lett., No. 3, 
August 9, 2016 19:55 WSPC/INSTRUCTION FILE SRI

Transient waves due to anti-symmetric seabed thrust 19

032005. 